\title{
Status of the KLOE-2 experiment
}

\author{
Danilo Domenici ${ }^{1}{ }^{\star \star}$ on behalf of the KLOE-2 Collaboration
}

${ }^{1}$ INFN - Laboratori Nazionali di Frascati, via E. Fermi 40, 00044 Frascati, Italy

\begin{abstract}
The KLOE-2 experiment is steadily taking data since November 2014 at DAFNE, the $\Phi$-factory in the Frascati National Laboratories. $3.5 \mathrm{fb}^{-1}$ of integrated luminosity have been collected up to now, with an average rate of $10 \mathrm{pb}^{-1} /$ day. Performance of the detector, data taking conditions and data quality monitoring are briefly summarized.
\end{abstract}

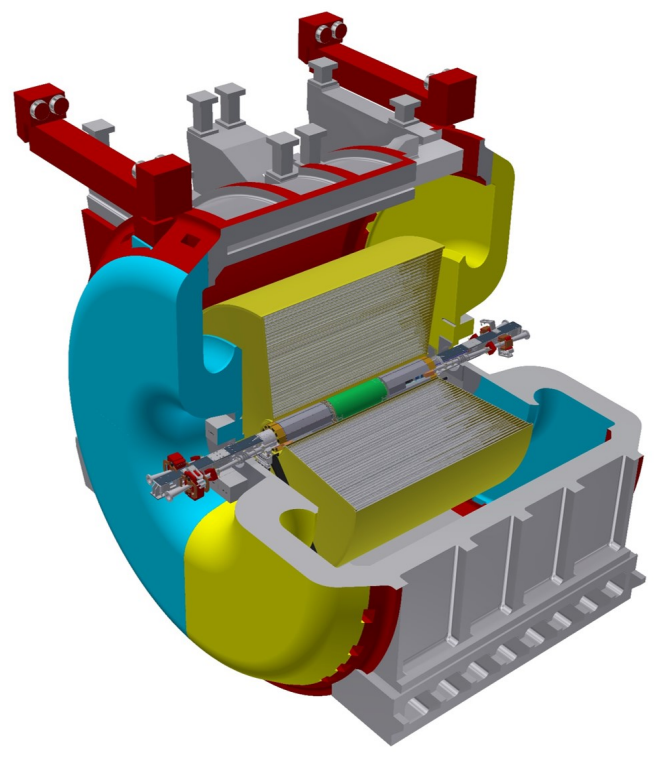

Figure 1. A sketch of the KLOE-2 detector.

\section{Introduction}

DAФNE (Double Annular $\Phi$-factory Nice Experiment) [1] is the last of a long tradition of electronpositron colliders realized at the Frascati National Laboratory of the Italian Institute for Nuclear Physics (INFN). It started its operations in 1999 and provided collisions for KLOE [2], DEAR [3]

^e-mail: Danilo.Domenici@lnf.infn.it 
and FINUDA [4] experiments until 2007. In this period KLOE collected $2.5 \mathrm{fb}^{-1}$ of integrated luminosity at $1019.4 \mathrm{MeV}$ of energy, corresponding to the $\Phi$ peak, and additional $250 \mathrm{pb}^{-1}$ at $1000 \mathrm{MeV}$ of energy.

In 2008 DAФNE has been upgraded with a deep change of the interaction region, implementing a large-Piwinski angle optics with crab waist, an innovative scheme developed in Frascati and tested here for the for the first time [5, 6]. Profiting from the improved performance in terms of luminosity, the KLOE detector has been upgraded with new detectors and started a second run as KLOE-2 at the end of 2014, with a refreshed physics program based on precise measurements achievable with DAФNE increased intensity [7]. The aim is indeed to collect $5 \mathrm{fb}^{-1}$ of integrated luminosity, enough to significantly increase the accuracy in the study, for instance, of $K_{S}, \eta$ and $\eta^{\prime}$ rare decays, as well as to set new limits on CPT symmetry violation and searches for hidden particles.

\section{The KLOE-2 detector}

KLOE-2 is general purpose detector, covering the full solid angle, composed by three main subdetector systems:

- The tracking system. The tracking is performed by a Drift Chamber (DC) and an Inner Tracker (IT) both immersed in an axial magnetic field of $0.52 \mathrm{~T}$ created by a superconductive coil.

- The calorimeter system. The central electromagnetic calorimeter (EMC) is composed by a barrel and two end-caps covering 98\% of the solid angle. The Quadrupole calorimeter (QCALT) instruments the two permanent focusing quadrupoles aside of the interaction point. The Crystal calorimeter (CCALT) has an acceptance for very low polar angles.

- The tagging system. There are a Low-energy tagger (LET) and a High-energy tagger (HET) devoted to identify events where photons are radiated by the beams.

\subsection{The tracking system}
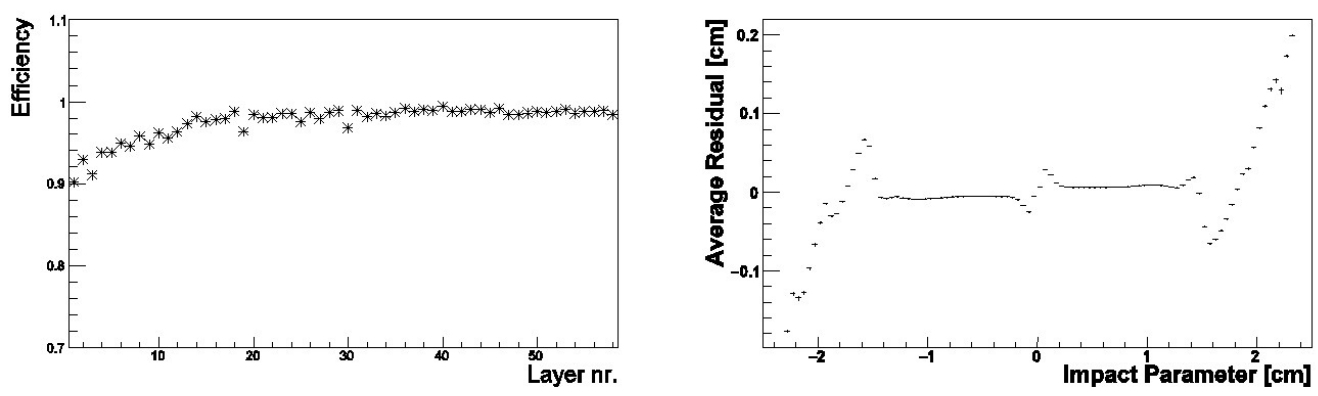

Figure 2. Performance of the Drift Chamber. Left: efficiency vs layer. Right: residuals vs impact parameter.

The KLOE Drift Chamber [8] is the largest detector of this kind ever built. It has a diameter of $4 \mathrm{~m}$ and a length of $3.7 \mathrm{~m}$, resulting in a huge volume of almost $50 \mathrm{~m}^{3}$ filled with a very light mixture of Helium:Isobuthane (90:10). The mechanical rigidity is provided by two plates of carbon fiber connected with 12000 sense wires and 40000 field wires creating a structure of 58 layers of drift cells with a stereo geometry allowing to extract information on the longitudinal coordinate as well. The 
spatial resolutions on the single hit are $\sigma_{\mathrm{r} \phi}=150 \mu \mathrm{m}$ and $\sigma_{\mathrm{Z}}=2 \mathrm{~mm}$ respectively for the bending and non-bending planes, while a decay vertex inside the detector volume is reconstructed with a resolution of $\sigma_{\mathrm{V}}=1 \mathrm{~mm}$.

The performance of the DC are continuously monitored using both cosmic rays muons and Bhabha scattering particles. Figure 2 shows the efficiency of the single layers and the residuals as a function of the track impact parameter.

The DAФNE beam-pipe around the interaction point is shaped as a sphere with a radius of $12 \mathrm{~cm}$, in order to keep most of the $K_{S}$ decays inside the vacuum and to avoid the $K_{L}$ regeneration rate depending by the emission angle. The innermost detecting layer of the DC is placed at $26 \mathrm{~cm}$ from the beam line. In order to reduce the lever arm and improve the resolution for reconstruction of vertexes near the interaction point, an Inner Tracker has been inserted inside the Drift Chamber. It is composed by four independent cylindrical layers of GEM detectors providing two-dimensional space point information with resolutions of $\sigma_{\mathrm{r} \phi}=250 \mu \mathrm{m}$ and $\sigma_{\mathrm{Z}}=400 \mu \mathrm{m}$ respectively for the bending and non-bending planes. The Inner Tracker and the Drift Chamber are used as an integrated tracking device, with a reconstruction strategy which starts from the long track in the DC and adds the four point in the IT implementing a Kalman Filter algorithm to update the track parameters. The innermost point placed at $13 \mathrm{~cm}$ from the beam line and the very good resolution in $\mathrm{Z}$ contribute to improve the resolution for $K_{S} \rightarrow \pi^{+} \pi^{-}$decay vertexes down to $\sim 0.5 \tau_{S}$.

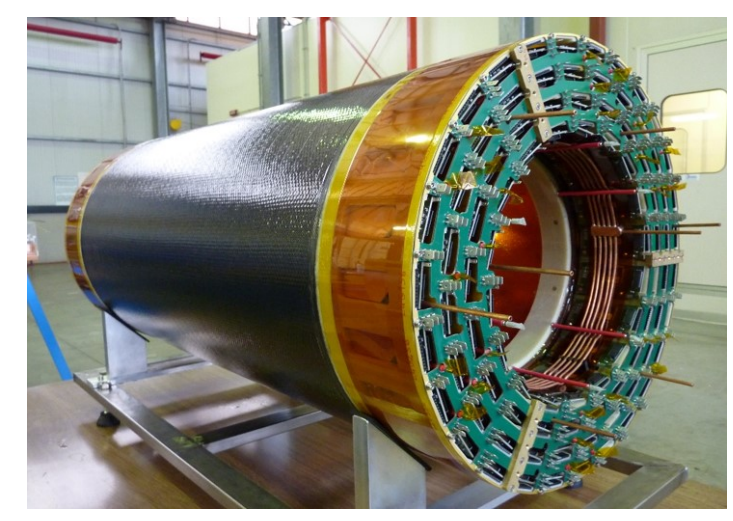

Figure 3. The KLOE-2 Inner Tracker before the insertion in the Drift Chamber.

The cylindrical GEM detectors have been realized by splicing and rolling single GEM foils with a technique specifically developed in Frascati [9]. The dead zones are thus reduced at minimum and the detector is very light, with an overall material budget corresponding to $2 \%$ of a radiation length, mandatory to minimize the multiple scattering, the photon conversion and the kaon regeneration. In order to achieve this result all the front-end electronics boards are placed at the two sides of the detector. The readout electrode has strips along the cylinder axis spaced with a pitch of $650 \mu \mathrm{m}$, straightforwardly providing the $\mathrm{r} \phi$ coordinate. The coordinate along the beam axis is obtained combining the $\mathrm{r} \phi$ coordinate with a $\mathrm{V}$ coordinate given by helicoidal strips crossing at an angle of $20^{\circ}$.

Figure 3 shows a preliminary distribution of the residuals for Bhabha scattering events reconstructed in DC and extrapolated to IT Layer 3 in $\mathrm{r} \phi$ coordinate. 


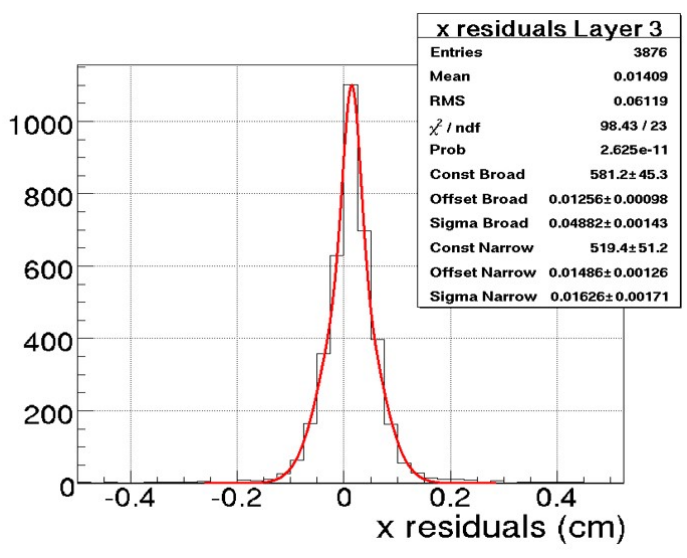

Figure 4. Residuals in $\mathrm{x}$ (corresponding to $\mathrm{r} \phi$ coordinate) of the Layer 3 of Inner Tracker with respect to DC tracks of Bhabha scattering events.

\subsection{The calorimeter system}

The KLOE electromagnetic calorimeter [10] is composed by a barrel and two end-caps with an hermeticity of $98 \%$ of the whole solid angle. The sampling structure is made by modules of five planes realized with a sandwich of scintillating fibers and $0.5 \mathrm{~mm}$ thin Lead foils. The resulting thickness of 15 radiation lengths allows a good efficiency also for neutral kaons and neutrons. The fibers are read-out by both sides of the modules by almost 5000 photo-multipliers coupled via light-guides.
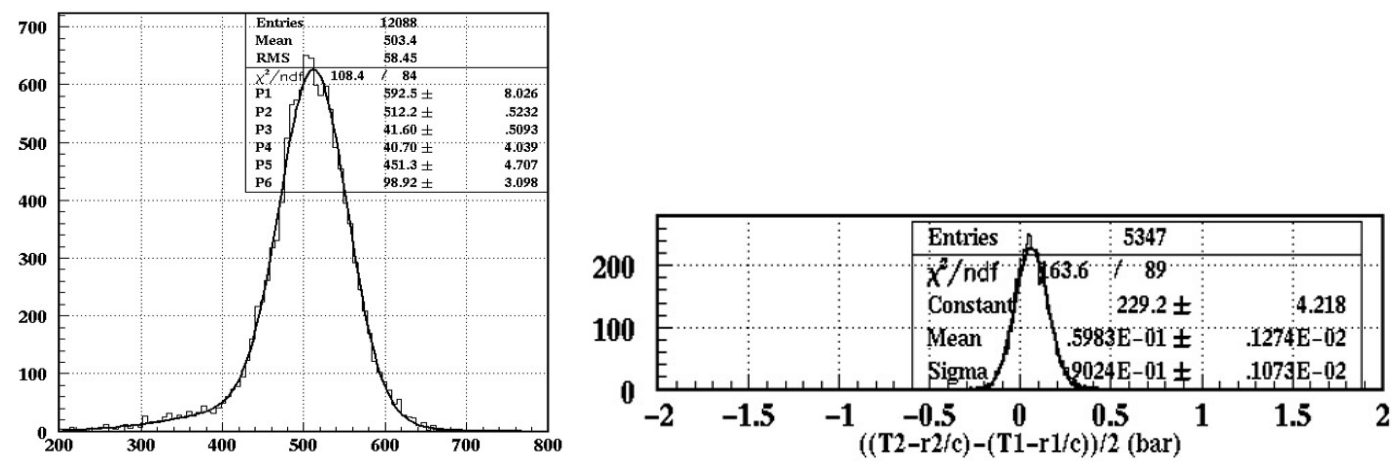

Figure 5. Performance of the Electromagnetic Calorimeter. Left: energy resolution. Right: time resolution.

Both energy and time resolutions (see Figure 5) are evaluated using $510 \mathrm{MeV}$ photons from $\gamma \gamma$ scattering events. The outstanding timing capabilities allow to perform particle identification with time of flight techniques.

At DAФNE the last focusing quadrupole are at only $1 \mathrm{~m}$ from the collision point, thus being placed inside the KLOE detector volume. In order to recover this space as an active area, the quadrupoles are surrounded by 2 structures made by 12 towers of Tungsten and scintillating tiles, the QCALT [11]. The light produced by the plastic scintillator is collected by wavelength-shifting fibers and read-out 
by Silicon Photo-multipliers. The QCALT is essential to increase the hermeticity of the calorimeter system, mostly for $K_{L}$ neutral decays.

To increase the acceptance for neutral decays of short-lived particles (e.g. $K_{S} \rightarrow \gamma \gamma$ ), two small calorimeters are placed just at $20 \mathrm{~cm}$ from the interaction point. Each CCALT is composed of 48 LYSO crystals directly coupled to a Silicon Photo-multiplier (SiPM) for the read-out. This detector can be also used as a luminosity monitor exploiting the abundant Bhabha scattering events at low angle and the good timing performance of the LYSO giving a good background rejection.

\subsection{The tagging system}

One of the main part of the KLOE-2 physics program is the study of mesons generated by a $\gamma \gamma$ fusion. The tagging of the events where both an electron and a positron undergo a bremsstrahlung emission is accomplished by two different systems [12]. Particles with an energy between 150 and $400 \mathrm{MeV}$ are detected by the Low Energy Taggers (LET), two stations placed at $1 \mathrm{~m}$ from the interaction point composed of 20 LYSO crystals read-out by SiPM. Particles with an energy above $400 \mathrm{MeV}$ are detected by the High Energy Taggers (HET), two plastic scintillator hodoscopes placed at $11 \mathrm{~m}$ from the interaction point, after the first bending dipoles of DAFNE.

\section{KLOE-2 data-taking}

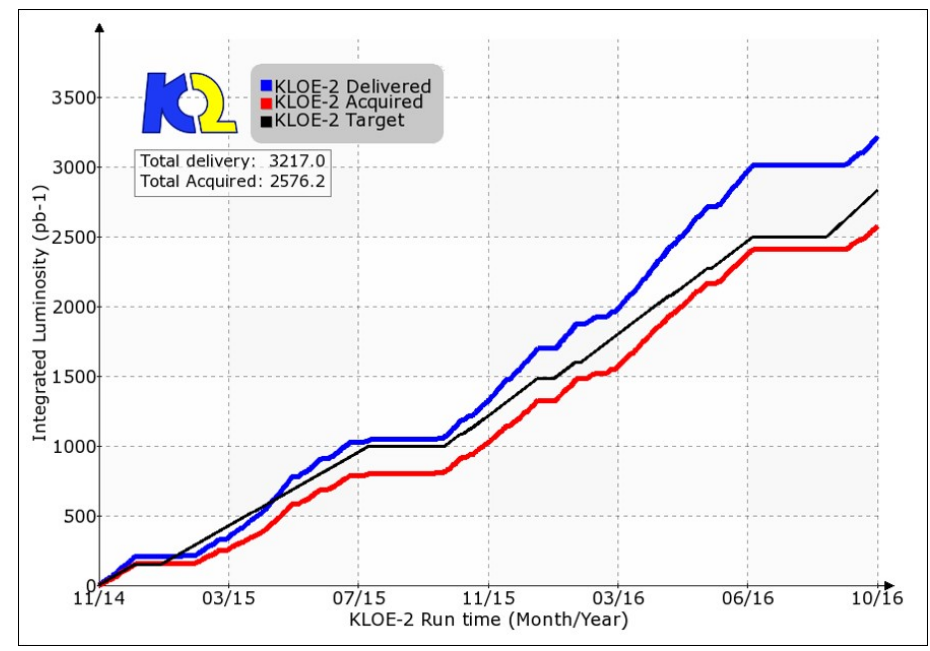

Figure 6. Summary of integrated luminosity of KLOE-2. Blue: delivered luminosity. Red: acquired luminosity. Black: goal for $5 \mathrm{fb}^{-1}$ delivery in March 2018.

Figure 6 shows the integrated luminosity of KLOE-2 starting from the beginning of the run, in November 2014. The $\sim 1 \mathrm{fb}^{-1}$ delivered in the first year has been doubled in the second year thanks to a deep optimization of the collider, and the progression is now steadily pointing towards the goal of $5 \mathrm{fb}^{-1}$ acquired by the end of the data-taking period, what required to accomplish our physics program.

The typical operational characteristics of DAFNE are: 
- beam currents of 1.5 A for electrons and $1 \mathrm{~A}$ for positrons

- 105 bunches stored with 2.7 ns spacing

- top-up injection of both beams every 10 minutes

Figure 7 shows beam currents and instantaneous luminosity during a typical hour of operation.

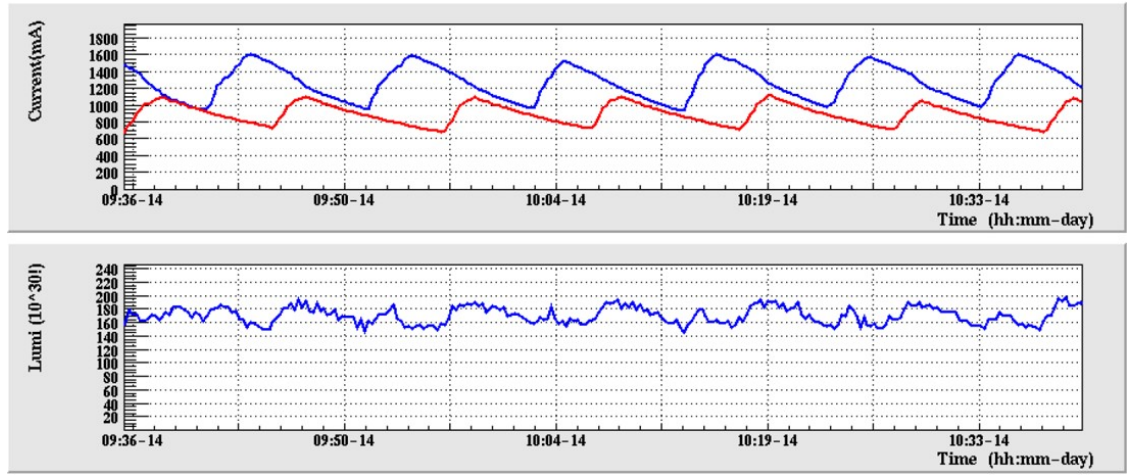

Figure 7. Currents and luminosity in a typical hour of collisions.

The best luminosity achievements of DAFNE in KLOE-2 data-taking are:

- $2.21 \times 10^{32} \mathrm{~cm}^{-2} \mathrm{~s}^{-1}$ as maximum instantaneous luminosity

- $651 \mathrm{nb}^{-1} \mathrm{~h}^{-1}$ as maximum hourly integrated luminosity

- $13.4 \mathrm{pb}^{-1} \mathrm{~d}^{-1}$ as maximum daily integrated luminosity

- $76.3 \mathrm{pb}^{-1} \mathrm{w}^{-1}$ as maximum weekly integrated luminosity

The KLOE-2 trigger is based on energy deposits in the Electromagnetic calorimeter. It requires two separated sectors with $\mathrm{E}>50 \mathrm{MeV}$ in the barrel and $\mathrm{E}>150 \mathrm{MeV}$ in the end-caps. At an average luminosity of $1.6 \times 10^{32} \mathrm{~cm}^{-2} \mathrm{~s}^{-1}$ the trigger rate is $7.5 \mathrm{kHz}$, with the following distribution:

- $500 \mathrm{~Hz}$ are due to $\Phi$ decays;

- $2.5 \mathrm{kHz}$ are due to Bhabha scattering events;

- $3 \mathrm{kHz}$ are due to cosmic rays;

- $1.5 \mathrm{kHz}$ are due to machine induced background, mostly Touschek scattering events, i.e. intra-bunch scattered particles.

\subsection{Data quality monitor}

Beam parameters like space position of the luminous point and transverse beam momentum are precisely measured with the KLOE detector and used as a monitor for the collider operation. The centerof-mass energy of the beams is also monitored using benchmark counters like e.g. $K_{S} \rightarrow \pi^{+} \pi^{-}$(see Figure 8) used:

In order to continuously monitor the quality of the collected data, four benchmark analysis are

- $\Phi \rightarrow \eta \gamma$ with $\eta \rightarrow 3 \pi^{0}$ 


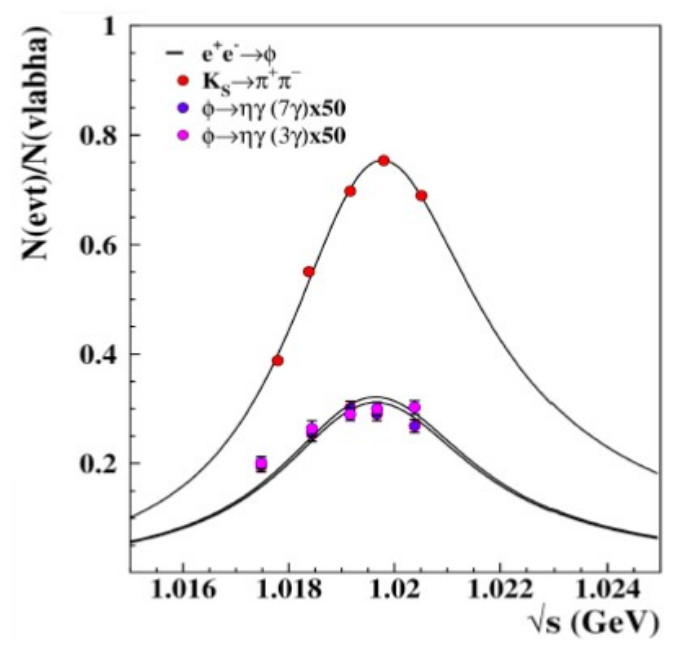

Figure 8. Scan of center-of-mass energy of the beams in steps of $550 \mathrm{keV}$ used to precisely set the operation point to the $\Phi$ mass peak at $1019.4 \mathrm{MeV}$.

- $\Phi \rightarrow \eta \gamma$ with $\eta \rightarrow \gamma \gamma$

- $K_{S} \rightarrow \pi^{+} \pi^{-}$

- $K_{L} \rightarrow \pi^{+} \pi^{-}$

The former are fully neutral channels useful to monitor mostly the performance of the calorimeter, while the latter are fully charged channels useful to monitor the performance of the tracking system.

\section{References}

[1] A. Gallo et al., Conf. Proc. C060626 (2006) 604, SLAC-PUB-12093

[2] KLOE Collaboration, Nuovo Cimento 30 (2008) 10

[3] DEAR Collaboration, Nucl. Phys. A 691 (2001) 278

[4] FINUDA Collaboration, Int. J. Mod. Phys. A 26 (2011) 420

[5] M. Zobov et al., Phys. Rev. Lett. 104, 174801 (2010).

[6] C. Milardi et al., JINST, 7, 2012,T03002.

[7] G. Amelino Camelia et al., Eur. Phys. J. C 68619 (2010)

[8] M. Adinolfi et al., Nucl. Inst. \& Meth. A 488 (2002) 51

[9] G. Bencivenni and D. Domenici, Nucl. Inst. \& Meth. A 581 (2007) 221

[10] M. Adinolfi et al., Nucl. Inst. \& Meth. A 482 (2002) 51

[11] F. Happacher and M. Martini, Acta Phys. Pol. B, 46 (2015) 87

[12] Babusci D. et al., Acta Phys. Pol. B, 46 (2015) 81 